\title{
Investigación en ingeniería en Colombia
}

\author{
Alberto Pertuz ${ }^{1 \mathrm{a}}$, Octavio Andrés González-Estrada ${ }^{1 \mathrm{~b}}$, Carlos Graciano $^{2}$ \\ ${ }^{1}$ Grupo de Investigación en energía y medio ambiente (Giema), Escuela de Ingeniería Mecánica, Universidad \\ Industrial de Santander, Colombia. Orcid: ${ }^{\mathrm{a}}$ 0000-0002-9130-6528, ${ }^{\mathrm{b}}$ 0000-0002-2778-3389. \\ Correos electrónicos: ${ }^{\mathrm{a}}$ apertuzc@ uis.edu.co, ${ }^{\mathrm{b}}$ agonzale@ saber.uis.edu.co \\ ${ }^{2}$ Departamento de Ingeniería Civil, Universidad Nacional de Colombia, Colombia. \\ Orcid: 0000-0003-0659-7963. Correo electrónico: cagracianog@unal.edu.co
}

Con el comienzo de una nueva década, se vislumbran fuertes cambios en muchos de los paradigmas de la ingeniería, y por supuesto, dichos cambios afectarán nuestra sociedad [1]. La aparición de tecnologías disruptivas como, por ejemplo, la inteligencia artificial, la manufactura aditiva, la realidad aumentada, plantean un nuevo escenario para la ingeniería, donde se fortalecerán los países que adopten oportunamente estas nuevas tecnologías. En este sentido, es importante hacer una valoración del estado actual de la investigación en el área de ingeniería en Colombia, y tratar de definir un escenario donde podamos hacer frente, de manera efectiva, a los nuevos retos que se plantean en esta nueva revolución industrial.

En la Figura 1 se muestran cifras referentes a la evolución de la investigación en el área de Ingeniería para Latinoamérica, según los registros de Scopus. En la Figura 1(a) se representa la producción científica para cada país vs. el número de publicaciones indexadas en Scopus [2] para el año 2018. Se observa la relación proporcional entre la producción y el número de revistas, siendo Brasil el primer país de la región con 18 revistas indexadas y 10363 documentos registrados. Colombia se ubica en el tercer lugar, con una proporción ligeramente más alta de revistas, con 6 revistas indexadas, y una producción de 2243 documentos. La Figura 1(b) muestra la evolución de la producción científica para los cuatro primeros países de la región entre los años 1996 y 2018 . Brasil ha sido el país dominante en el área de Ingeniería, con una producción total de 115610 documentos y un índice de impacto $h=205$. Colombia ocupaba el cuarto lugar en la región hasta el 2009, año en que superó a Chile. Hoy en día Colombia continua en el tercer lugar, con una producción total de 16678 documentos y un índice $h=94$ para el mismo período. Estos resultados muestran un avance positivo en la investigación en el área de Ingeniería para el país, especialmente si tenemos en cuenta que, según datos del Banco Mundial [3], Brasil invierte 1.27\% de su PIB en investigación y desarrollo, mientras que Colombia quedaría de cuarto en este mismo grupo, con una inversión del $0.24 \%$ del PIB.

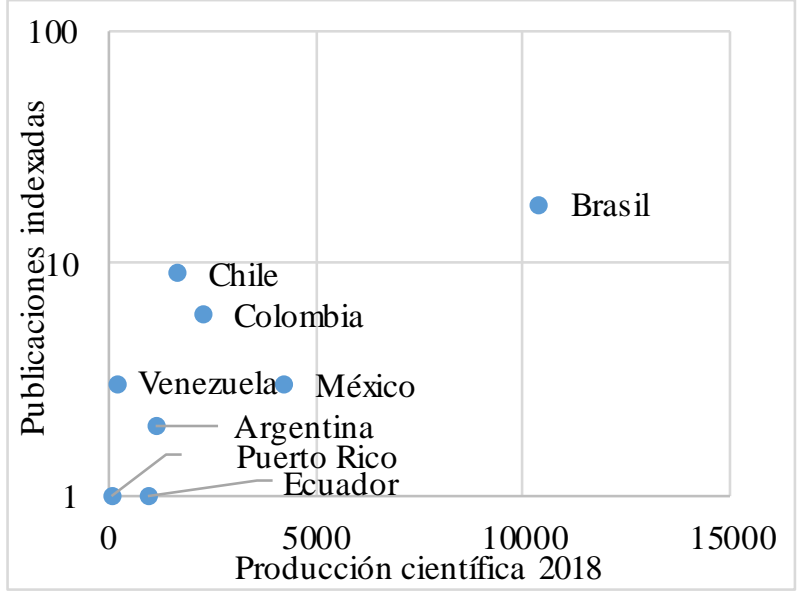

(a)

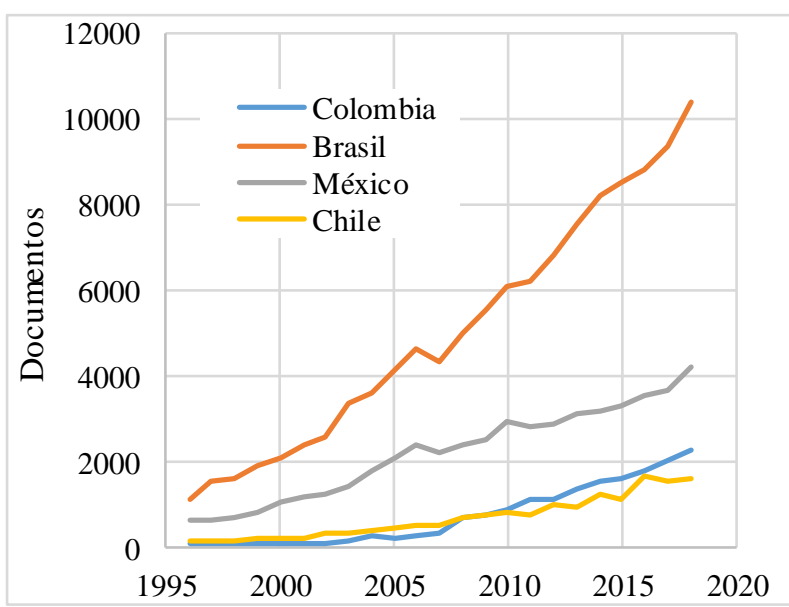

(b)

Figura 1. Producción en investigación en el área de Ingeniería para Latinoamérica (Scopus): (a) Producción científica vs. Número de publicaciones indexadas en Scopus, (b) Evolución del número de documentos por año para los cuatro primeros países de la región. Fuente: elaboración propia.

Se realizó la búsqueda en Scopus de la producción científica colombiana en el área de Ingeniería entre los años 2015 y 2020, para un total de 10125 documentos [4]. Posteriormente, se analizó de la información con la herramienta

ISSN impreso: 1657 - 4583. ISSN en línea: 2145 - 8456, CC BY-ND 4.0 cc) ( )

A. Pertuz, O. A. González-Estrada, C. Graciano, "Editorial-Investigación en ingeniería en Colombia,” Rev. UIS Ing., vol. 19, no. 1, pp. 7-14, 2020. doi: 10.18273/revuin.v18n4-2020021 
bibliométrica VOSviewer [5]. En la Figura 2 se muestra el mapa de co-ocurrencia de las palabras clave indexadas por la base de datos para los documentos en el área de Ingeniería. Dentro de la red generada se observan tres clústeres de investigación principales: métodos numéricos, ciencia de los materiales y energía.

En temas relacionados con métodos numéricos destacan mecánica de fluidos computacional y el método de los elementos finitos, los cuales corresponden a áreas de investigación bien establecidas. Sin embargo, también se observa gran crecimiento en temas de actualidad como machine learning y procesamiento de imagen. Todos estos temas están enmarcados dentro de una nueva disciplina que la National Science Foundation (NSF) ha denominado Simulationbased Engineering Science (SBES), la cual es un campo interdisciplinar que combina los conocimientos de diferentes campos tradicionales de la ingeniería con los conocimientos de campos como la ciencia de la computación, matemáticas y física [6]. La SBES plantea nuevos retos para la solución de problemas cada vez más complejos, como p. ej., el modelado multiescala, el uso de enfoques probabilísticos para el tratamiento de la información, el modelado de sistemas basados en datos dinámicos, el aprovechamiento de la información por medio del uso de big data y high performance computing (HPC). Cabe destacar que el área de SBES tiene una ventaja diferencial respecto a otras áreas, y ofrece una oportunidad de crecimiento para el país, ya que no demanda los altos niveles de inversión requeridos por las áreas experimentales.

En el clúster de ciencias de los materiales se destacan técnicas de caracterización de propiedades mecánicas e investigación dirigida al sector de la construcción. En la mayoría de los países, la ciencia de los materiales ha sido un área de investigación tradicional. Hoy en día, la tendencia en ingeniería sigue planteando la búsqueda y diseño de nuevos materiales, con propiedades singulares como, p. ej., los metamateriales, sintetizados o formados en diferentes escalas gracias a la nanofabricación [7], o materiales para aplicaciones en áreas como la salud o la energía.

Por otro lado, en el tercer clúster se observan tendencias mundiales, como es, por ejemplo, la investigación dirigida al desarrollo sostenible. En su visión para 2030, las Naciones Unidas ha planteado retos en torno al desarrollo sostenible dirigidos a plantear una transformación de la sociedad, la integridad medioambiental y la viabilidad económica. En este sentido, se puede observar la tendencia en ingeniería hacia la investigación en áreas como eficiencia energética, biomasa y desarrollo sostenible.

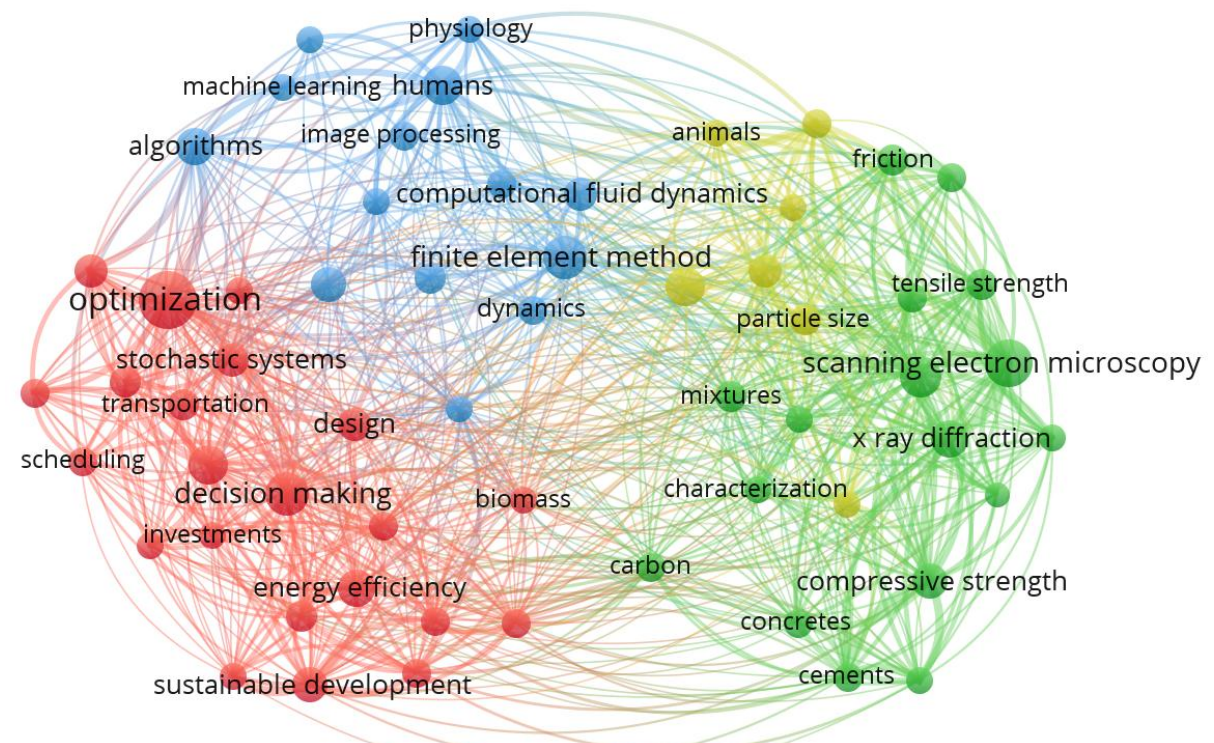

Figura 2. Mapa de co-ocurrencia de palabras clave en la producción científica para el área de Ingeniería. Fuente: elaboración propia.

La Figura 3 muestra el mapa de co-ocurrencia en el tiempo de palabras clave en la producción científica de mayor impacto en el área de Ingeniería, donde el impacto se mide a partir del número de citas de cada documento en la base de datos Scopus. Para la extracción de la información se ha considerado el período 2000-2020, y se han tomado los 2000 documentos con mayor número de citas. Este mapa nos ofrece una idea de la evolución de los temas de investigación en el país, mostrando las nuevas tendencias y áreas de mayor maduración. En el mapa se muestra el impacto de los trabajos desarrollados en el área de estructuras: buckling, stability, columns, nonlinear analysis, etc., la 
cual se observa lleva un tiempo más largo de permanencia. Aparecen nuevamente, con un alto impacto, los trabajos desarrollados en el área de ciencias de los materiales, donde se pueden observar temas de amplia tradición en el país como, p. ej., la caracterización y corrosión de materiales, y temas más recientes en nanotecnología y nuevos materiales como el grafeno. Igualmente, en el área de energía, se observan áreas de actual crecimiento como la investigación en biomasa, sostenibilidad y eficiencia energética.

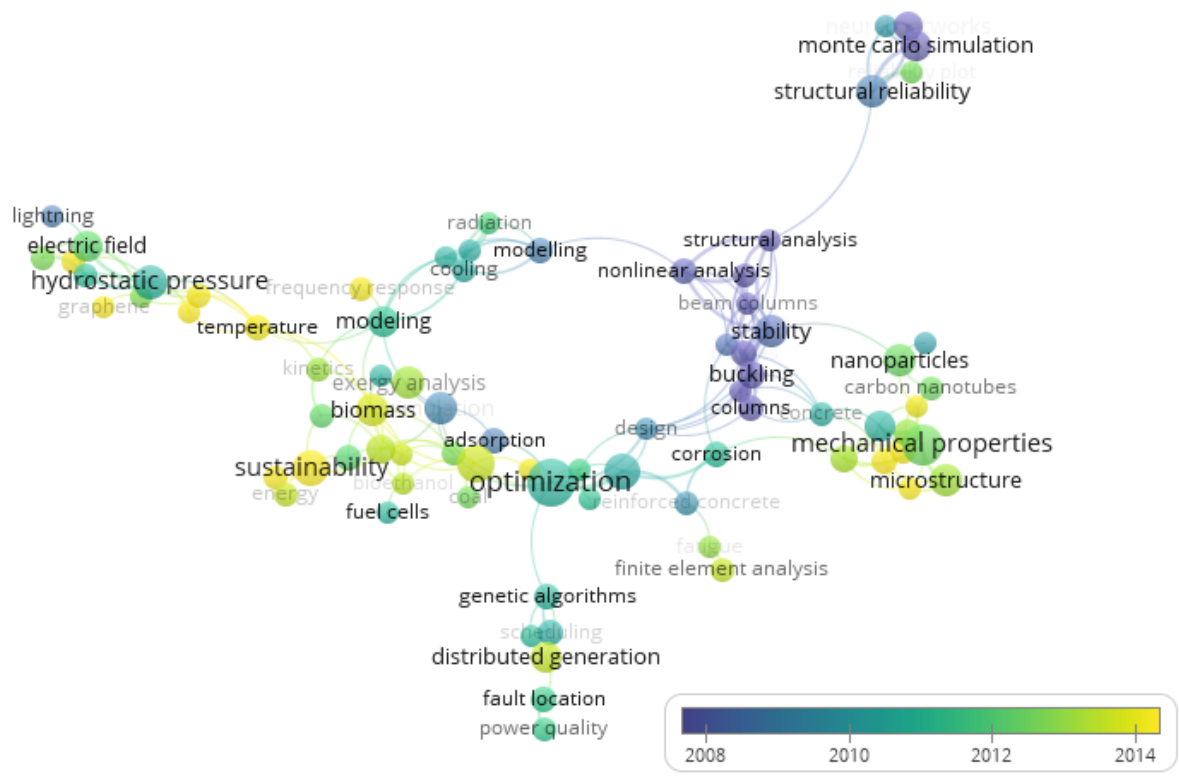

Figura 3. Mapa de co-ocurrencia en el tiempo de palabras clave en la producción científica de mayor impacto para el área de Ingeniería de 2000 a 2020. Fuente: elaboración propia.

Por otra parte, en la Figura 4 se muestra la red de colaboración entre países obtenida a partir del análisis de la información bibliométrica de los documentos en el área de Ingeniería. Destacan en la red la colaboración con Estados Unidos, España y Brasil. Asimismo, dentro de la red se observa el clúster de países iberoamericanos. También se observa en el mapa una amplia red de países cuyas colaboraciones indirectas resultan de la participación en redes y grupos de investigación transnacionales, derivado de los procesos de globalización de la ciencia.

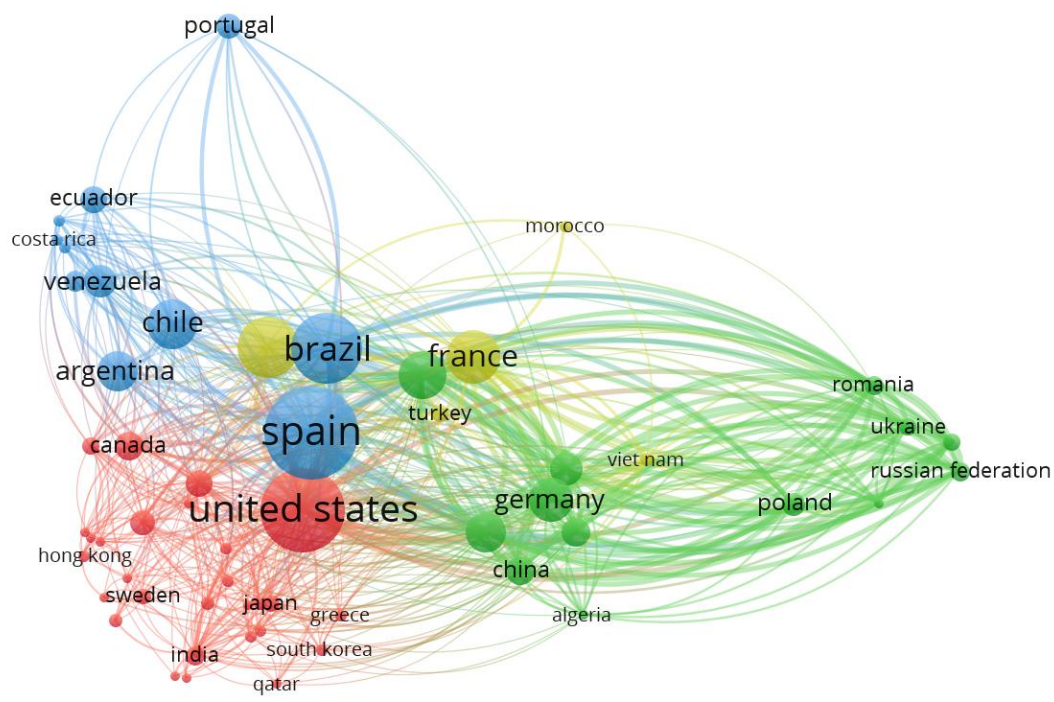

Figura 4. Mapa bibliométrico de la colaboración con otros países a partir de la producción científica para el área de Ingeniería en Colombia. Fuente: elaboración propia. 
En conclusión, se observa un crecimiento sostenido de la investigación en el área de ingeniería, apoyado por la internacionalización de la ciencia y que, con un gran esfuerzo, ha tratado de suplir las falencias asociadas a la falta de inversión en ciencia y tecnología en Colombia. La producción científica ha aumentado en cantidad, en parte gracias a la gestión del conocimiento basada en el capitalismo académico [8], [9], y el reto se plantea ahora en mejorar la calidad y el impacto de la investigación realizada. En este sentido, resulta importante dirigir las políticas de inversión en ciencia y tecnología hacia la investigación aplicada, de manera que nos permita abordar problemas asociados a los retos en desarrollo sostenible y la transformación de la sociedad.

\section{Referencias}

[1] H. Lasi, P. Fettke, H.-G. Kemper, T. Feld, and M. Hoffmann, "Industry 4.0," Bus. Inf. Syst. Eng., vol. 6, no. 4, pp. 239-242, Aug. 2014.

[2] S. Lab, "SJR Visualization Tools," 2020 [En línea]. Disponible en: https://www.scimagojr.com/viztools.php.

[3] Grupo Banco Mundial, "Gasto en investigación y desarrollo (\% del PIB) Data,” 2019. [En línea]. Disponible en:: https://datos.bancomundial.org/indicador/GB.XPD.RSDV.GD.ZS.

[4] Elsevier B.V., "Scopus,” 2020. [En línea]. Disponible en: Available: http://www.scopus.com/

[5] N. J. van Eck and L. Waltman, "Software survey: VOSviewer, a computer program for bibliometric mapping," Scientometrics, vol. 84, no. 2, pp. 523-538, Aug. 2010.

[6] M. Stojek and J. Pietraszek, "Simulation-Based Engineering Science Challenges of the 21st Century," Appl. Mech. Mater., vol. 712, pp. 3-8, 2015.

[7] C. A. Garzón and C. E. Moreno, "La facultad de ingeniería 2030,” Ing. e Investig., vol. 31, no. 1, pp. 91-99, 2011.

[8] I. C. Montes and P. Mendoza, "Docencia e investigación en Colombia desde la perspectiva del capitalismo académico," Educ. Policy Anal. Arch., vol. 26, no. 40, pp. 1-33, 2018.

[9] G. Rueda-Barrios and M. Rodenes-Adam, "Factores determinantes en la producción científica de los grupos de investigación en Colombia," Rev. Española Doc. Científica, vol. 39, no. 1, pp. 1-16, 2016. 